FEATURE

\title{
SEATURE
}




\section{Medical Mimics of Psychiatric Conditions, Part 1}

Steven R. Knight, MD; Mary Nan S. Mallory, MD, MBA; Martin R. Huecker, MD

\section{In part 1 of this 2-part series, the authors review medical conditions with infectious, pharmacological, metabolic, autoimmune, traumatic, or CNS causes that can present as behavioral or psychiatric emergencies.}

T he chaos of a busy ED can test the cognitive reserve of even the most focused practitioner. To streamline the challenge of serial diagnosis and treatment, clinicians employ heuristics while honing the skills of pattern recognition. However, by definition, heuristics employs shortcuts, leaving out information for the sake of efficiency-sometimes at the expense of accuracy. Whether a patient presents with chest pain, abdominal pain, headache, or (the dreaded) dizziness, emergency physicians (EPs) employ algorithms based on a combination of education and prior experience.

Most of the time, these models lead the EP along the correct path, but not always. For example, when a clinician evaluating a patient presenting with psychotic behavior assumes the patient has schizophrenia, he or she will be correct eight or nine times out of 10. However, in some cases, a patient's bizarre behavior may not be due to a true psychiatric disorder but, for example, from ingestion of an illicit substance.

In addition, in such patients, psychiatric symptoms may be masking a serious acute, organic condition-one requiring prompt intervention and therapy to avoid morbidity or death. To help prevent diagnostic errors, this 2-part series reviews several of the most common medical mimics of psychiatric conditions. Part 1 of this series reviews the psychiatric presentations associated with medical conditions of an infectious, pharmacological withdrawal, metabolic, autoimmune, traumatic, or central nervous system etiology (Table 1). This article also discusses clinical signs and symptoms that suggest an increased likelihood that a patient's psychiatric symptoms are from an underlying medical condition (Table 2).

\section{Case Scenarios}

\section{Case 1}

A 58-year-old woman with a history of smoking 40 packs of cigarettes per year presented to the ED 1 hour after onset of intermittent chest pain. Upon arrival at the ED, the patient stated that she had trouble catching her breath on and off throughout the day. The patient's vital signs, electro-

Dr Knight is an emergency medicine resident, University of Louisville School of Medicine, Kentucky. Dr Mallory is a professor of emergency medicine at the University of Louisville School of Medicine, Kentucky. Dr Huecker is an assistant professor, Department of Emergency Medicine, University of Louisville School of Medicine, Kentucky.

DOI: 10.12788/emed.2016.0026 
Table 1. Psychiatric Presentations Associated WIth Medical Etiologies

\begin{tabular}{|c|c|c|}
\hline Etiology & Diagnosis & Psychiatric Presentation \\
\hline \multirow[t]{5}{*}{ Infectious } & Herpes simplex encephalitis ${ }^{1}$ & $\begin{array}{l}\text { Behavioral changes, cognitive decline, aggression, } \\
\text { disinhibition }\end{array}$ \\
\hline & Human immunodeficiency virus ${ }^{2}$ & Early-onset, rapidly progressive dementia \\
\hline & Progressive multifocal encephalopathy ${ }^{3}$ & $\begin{array}{l}\text { Clumsiness, weakness, visual changes, speech difficulty, } \\
\text { behavioral changes }\end{array}$ \\
\hline & Syphilis 4,5 & Personality disorder, psychosis, delirium, dementia \\
\hline & $\begin{array}{l}\text { Typhoid fever } \\
\text { and other endemics }\end{array}$ & Acute confusion, psychosis, anxiety, depression (rare) \\
\hline \multirow[t]{7}{*}{$\begin{array}{l}\text { Pharmacological } \\
\text { withdrawal }\end{array}$} & Alcohol ${ }^{7,8}$ & $\begin{array}{l}\text { Agitation, hallucinations, persecutory delusions, } \\
\text { self-mutilation }\end{array}$ \\
\hline & \multirow[t]{2}{*}{ Benzodiazepines ${ }^{9,10}$} & $\begin{array}{l}\text { Therapeutic doses: sleep disturbances, irritability, } \\
\text { anxiety, panic attacks, perceptual changes }\end{array}$ \\
\hline & & Larger doses: acute psychosis \\
\hline & Opiates $^{11}$ & Anxiety, agitation, irritability \\
\hline & \multirow[t]{2}{*}{ Cannabis $^{12}$} & Type 1: weakness and hypersomnia \\
\hline & & Type 2: anxiety, depression, restlessness, and insomnia \\
\hline & Estrogen ${ }^{13}$ & Acute psychosis \\
\hline \multirow[t]{2}{*}{ Acute metabolic } & Hypoglycemia $^{14}$ & Confusion, anxiety, nervousness, nightmares \\
\hline & Central pontine myelinolysis ${ }^{15}$ & Behavioral changes, psychosis, cognitive disturbances \\
\hline \multirow[t]{3}{*}{ Autoimmune } & Systemic lupus erythematosus ${ }^{16}$ & $\begin{array}{l}\text { Cognitive dysfunction, mood disorders, acute confusion, } \\
\text { psychosis, paranoia, auditory or visual hallucinations }\end{array}$ \\
\hline & $\begin{array}{l}\text { Anti-N-methyl-D-aspartate receptor } \\
\text { encephalitis }\end{array}$ & $\begin{array}{l}\text { Acute psychosis, delusional thinking, hallucinations, } \\
\text { agitation, confusion }\end{array}$ \\
\hline & Multiple sclerosis ${ }^{20}$ & $\begin{array}{l}\text { Anxiety, depression, acute psychosis, adult-onset } \\
\text { tic disorder }\end{array}$ \\
\hline \multirow[t]{2}{*}{ Traumatic } & Subarachnoid hemorrhage ${ }^{21}$ & $\begin{array}{l}\text { Personality changes, intellectual impairment, mood } \\
\text { disorders, kleptomania, akinetic mutism, confabulatory } \\
\text { amnesia, acute psychosis, Capgras syndrome }\end{array}$ \\
\hline & Subdural hematoma ${ }^{22}$ & $\begin{array}{l}\text { Cognitive impairment, withdrawn behavior, blunted } \\
\text { affect, catatonia }\end{array}$ \\
\hline \multirow{4}{*}{$\begin{array}{l}\text { Central nervous } \\
\text { system disease }\end{array}$} & Huntington disease ${ }^{21}$ & Episodic psychosis \\
\hline & Parkinson disease ${ }^{22}$ & $\begin{array}{l}\text { Episodic psychosis, visual hallucinations, mood } \\
\text { disorders, apathy, executive dysfunction }\end{array}$ \\
\hline & Temporal lobe epilepsy²3 & $\begin{array}{l}\text { Depression, fear, anxiety, recurrent déjà vu, flashbacks, } \\
\text { paranoid delusions, depersonalization, derealization }\end{array}$ \\
\hline & Stroke ${ }^{24}$ & $\begin{array}{l}\text { Confusion, altered mental status, etc; positive predictive } \\
\text { value of } 7 \% \text { for altered mental status alone }\end{array}$ \\
\hline
\end{tabular}


cardiogram (ECG), and chest X-ray were all normal. The physical examination was unremarkable except for mild diaphoresis. The patient denied experiencing palpitations, recent travel, or previous episodes; she further stated that she was currently not on any medications. There was no previous history of visits to this hospital. The patient's husband, who accompanied her to the ED, noted that his wife's behavior had been atypical for approximately 1 week.

After receiving aspirin, the patient appeared symptom-free. Pending the results of another chest radiograph and laboratory evaluation, the EP anticipated moving her to the chest-pain observation unit.

\section{Case 2}

A 36-year-old woman presented with altered mental status to the ED via emergency medical services (EMS). Her vital signs, including temperature, were normal. Despite intermittently appearing to be asleep, the patient was alternatingly cooperative and combative. She repetitively whispered, "Who am I?" and randomly shouted at staff members as they walked by her room.

Her neurological examination was nonfocal. The hospital's electronic medical record (EMR) for this patient showed nearly monthly ED visits for behavioral symptoms. Precipitating events noted in the EMR included job loss and separation from her husband. While waiting for the results of the basic laboratory work-up and toxicology screening to medically clear the patient for psychiatric evaluation, the EP contemplated a computed tomography (CT) study. Realizing the patient would not be able to remain still for the scan, the EP ordered 10 mg of intramuscular ziprasidone for sedation. When the patient's husband arrived, the EP placed the CT scan on hold until she could obtain additional history from him.

\section{Infections}

\section{Herpes Simplex Encephalitis}

Herpes simplex encephalitis (HSE) is a serious but treatable disease-one that requires
Table 2. A Summary of High-risk Features for an Underlying Medical Mimic

Over age 40 years with no previous psychiatric history

No history of similar symptoms, or worsening of previous symptoms

Family concern (eg, "This is not his/her usual behavior")

Chronic disease comorbidities

History of head injury

Change in headache pattern

Patient worse after antipsychotic or anxiolytic

Difficult or unlikable patient

History of changing psychiatric diagnoses over time

Polypharmacy

Abnormal autonomic signs

Visual disturbance (field loss or double vision)

Visual, olfactory, or tactile hallucinations

Nystagmus (often with drug intoxications)

Illusions

Speech deficit (dysarthria or aphasia)

Abnormal body movement

early detection and treatment to avoid severe morbidity. While the classic symptoms are fever and altered mental status, recent literature has noted that afebrile patients with HSE may present with behavioral changes, cognitive decline, aggression, and disinhibition. Therefore, diagnosis of a functional psychiatric complaint, if made initially, could delay appropriate treatment with acyclovir. ${ }^{1}$

\section{Human Immunodeficiency Virus}

Progression of human immunodeficiency virus (HIV) is a wellknown cause of various neurocognitive disorders, including early-onset dementia. Since the availability of highly active antiretroviral therapy, the incidence of HIV dementia has decreased, but HIV remains the most common preventable cause 
of dementia in persons younger than age 50 years. Recent literature has described HIV dementia presenting as an early-onset, rapidly progressing dementia in a young person. Thus, the EP should consider early HIV testing in any young patient who presents with dementia, especially one with a history of fever of unknown origin. ${ }^{2}$

\section{Progressive Multifocal Encephalopathy}

Caused by reactivation of the John Cunningham virus, progressive multifocal encephalopathy has been classically described as a potentially lethal complication of a severely immunocompromised state, often presenting with clumsiness, weakness, visual changes, speech difficulty, and behavioral changes. Though typically described as occurring in the context of acquired immunodeficiency disease syndrome, hematological malignancy, or organ transplant, the condition can occur in the setting of minimal or occult immunosuppression-especially in patients with a history of cirrhosis. If the condition is detected early, immunotherapy can result in significant clinical improvement. ${ }^{3}$

\section{Syphilis}

Late stages of syphilis can present with a wide variety of psychiatric symptoms, including personality disorder, psychosis, delirium, and dementia. As with HIV, there has been a resurgence of syphilis cases, and screening is now often a routine part of a neuropsychiatric work-up. The EP should consider syphilis in the differential for any new-onset psychiatric complaint. ${ }^{4,5}$

\section{Typhoid Fever}

Although this severe febrile illness is uncommon in the United States, it is endemic to many tropical countries within Africa, Southeast Asia, and Central and South America. Typhoid is characterized by a stepwise fever that can progress to abdominal distension, toxemia, and potentially bowel perforation. It is also known to present with psychiatric symptoms such as acute confusion, psychosis, generalized anxiety disorder, and, though rare, depressive disorder. Physicians traveling to rural endemic areas should be aware of these neuropsychiatric presentations to avoid misdiagnosis and delay of treatment. ${ }^{6}$ Other infectious endemic diseases with reports of neuropsychiatric components are neurocystercercosis, Lyme disease, and African trypanosomiasis.

\section{Pharmacological Withdrawal Syndromes \\ Alcohol}

Alcohol withdrawal is a common presentation in the ED, and up to $24 \%$ of US adults brought to the ED by EMS suffer from alcoholism. Typically characterized by tachycardia, hypertension, and tremors, alcohol withdrawal syndrome can also feature psychiatric components such as agitation, hallucinations, persecutory delusions, and even self-mutilation. ${ }^{7}$ Evidence-based protocols indicate loading doses of benzodiazepines as a mainstay of treatment, with supplemental barbiturates or propofol in cases of treatment failure. ${ }^{8}$

\section{Benzodiazepines}

Withdrawal from therapeutic doses of benzodiazepines can potentially cause psychiatric symptoms, including sleep disturbances, irritability, anxiety, panic attacks, tremor, and perceptual changes. Withdrawal from higher doses of benzodiazepines can lead to more serious presentations, such as seizures and acute psychosis. ${ }^{9}$ Withdrawal symptoms can develop from discontinuation of the drug and with non-tapered switching between benzodiazepines. ${ }^{10}$

\section{Opiates}

Opiate withdrawal is an unpleasant experience characterized by generalized pain, nausea and vomiting, sweating, and tachy- 
cardia. Neuropsychiatric complaints such as anxiety, agitation, and irritability can also be present. More severe agitation has been described in naltrexone-accelerated detoxification $^{11}$

\section{Cannabis}

Recent literature on cannabis use indicates a high prevalence and clinical significance of associated withdrawal symptoms in frequent users. There appear to be two subsets of cannabis withdrawal-one characterized by weakness and hypersomnia, and the other by anxiety, depression, restlessness, and insomnia. ${ }^{12}$

\section{Estrogen}

Withdrawal from endogenous estrogen has been hypothesized as a possible cause of puerperal psychosis. ${ }^{13}$ Estrogen withdrawal outside of this setting, however, can and does occur, and recent literature has shown episodes of reversible psychosis associated with the discontinuation of both oral contraceptive regimens and hormonal therapy for menopausal symptoms.

\section{Acute Metabolic Conditions Hypoglycemia}

Hypoglycemia, most often encountered as a side effect of insulin or oral hypoglycemic therapies, is a potentially lethal cause of confusion, anxiety, nervousness, and seizures. Nocturnal hypoglycemia can manifest as nightmares, crying out, and confusion upon awakening. A fingerstick blood-glucose test is an absolutely vital part of the initial work-up of any patient with an altered mental status or overt psychiatric complaint. ${ }^{14}$

\section{Central Pontine Myelinolysis}

A potentially devastating neurological condition associated with malnourishment and alcohol dependence, central pontine myelinolysis (CPM) is classically exacerbated by rapid overcorrection of hyponatremia. While the disease can manifest primarily with quadriplegia or pseudobulbar palsy and eventual progress to the dreaded "locked-in" syndrome, early presentations can include psychiatric symptoms such as behavioral changes, psychosis, and cognitive disturbances. Patients with early signs and symptoms of CPM have been misdiagnosed as having schizophrenia with catatonia, leading to delayed treatment and poor outcomes. The EP should remain vigilant when evaluating for this condition and consider a magnetic resonance imaging study in patients with psychiatric symptoms in the setting of fluctuating hyponatremia. ${ }^{15}$

\section{Autoimmune Disorders}

\section{Systemic Lupus Erythematosus}

Systemic lupus erythematosus (SLE) is one of the most common autoimmune disorders, and has a higher incidence in young women. The disease affects multiple organ systems. Though the classic initial presentation of SLE is fever, joint pain, and rash, the associated neuropsychiatric syndromes of this disease are diverse and surprisingly common, and can be the initial manifestation of the disease. Common psychiatric manifestations of SLE include cognitive dysfunction, anxiety, mood disorders such as depression, acute confusion, psychosis, paranoia, and auditory or visual hallucinations. ${ }^{16}$

\section{Anti-N-methyl-D-Aspartate Receptor Encephalitis}

Initially described as a paraneoplastic effect of ovarian teratomas, anti-N-methyl-Daspartate receptor (anti-NMDAR) encephalitis is actually an autoimmune disorder that can occur even in the absence of a primary tumor. As with SLE, the condition primarily occurs in young women. Antibodies in the cerebrospinal fluid cause prominent psychiatric symptoms such as acute psychosis, delusional thinking, hallucinations, agitation, and confusion. Although the disease can progress to seizures, 
movement disorders, autonomic dysregulation, and ultimately death, early recognition and treatment can lead to positive outcomes in up to $80 \%$ of cases. ${ }^{17}$ While the prevalence of anti-NMDAR antibodies in new-onset psychosis remains unclear, recent literature has suggested widespread screening for the disease in all first presentations of psychotic episodes. ${ }^{18,19}$

\section{Multiple Sclerosis}

Multiple sclerosis (MS) is another autoimmune disorder that has a higher prevalence in young women. The disease is characterized by central nervous system involvement that occurs over a period of months to years, with symptoms corresponding to different anatomic locations. Though the classic presenting symptom of MS is optic neuritis, neuropsychiatric syndromes are a common co-occurrence and can be the initial presenting symptom. The most commonly associated psychiatric complaints are anxiety, depression, and bipolar disorder, though case reports of SLE have described acute psychosis, psychotic depression, and adult-onset tic disorder. ${ }^{20}$

\section{Trauma}

\section{Subarachnoid Hemorrhage}

Long-term psychiatric sequelae from subarachnoid hemorrhage, either traumatic or aneurysmal, manifest most commonly as personality changes, intellectual impairment, depression, and anxiety. This condition is also known to cause a host of more bizarre psychiatric presentations, such as new-onset kleptomania, akinetic mutism, confabulatory amnesia, acute psychosis, and Capgras syndrome (the delusion that familiar individuals have been replaced by imposters). These symptoms can occur at initial presentation, and may show variable improvement with shunt surgery. ${ }^{21}$

\section{Subdural Hematoma}

Acute or chronic subdural hematoma can result from major head trauma, or even quite minor head trauma in an elderly or coagulopathic patient. Some common psychiatric manifestations of subdural hematoma include cognitive impairment, withdrawn behavior, blunted affect otherwise mimicking schizophrenic psychosis, and catatonia. The EP should consider early imaging studies in patients with new-onset psychotic symptoms-especially when they are refractory to typical antipsychotics. ${ }^{22}$

\section{Central Nervous Symptom Diseases \\ Huntington Disease}

Huntington disease (HD) is an autosomal dominant inherited, progressive neurodegenerative disorder characterized by mental decline, mood disorder, and muscle coordination problems that eventually become the classic involuntary writhing termed chorea. Due to its progressive nature, precise onset of the disease is difficult to describe; however, HD can manifest initially as schizophrenia-like psychotic episodes with only minimal apparent motor difficulty. Family history, including movement disorders and suicide, is important to obtain when available. ${ }^{23}$

\section{Parkinson Disease}

A progressive and disabling neurodegenerative disorder, Parkinson disease (PD) is classically characterized by fine resting tremor, cogwheeling rigidity, akinesia and mask-like facies, and postural instability. Comorbidity of psychiatric disorders is high, both as a result of the underlying disease process and as a side effect of dopaminergic treatment regimes. Common presentations of psychiatric disorders in $\mathrm{PD}$ include schizophrenia-like psychosis with visual hallucinations and mood disorders with prominent apathy and executive dysfunction. Recognition of the comorbidity is important because psychiatric disorders in PD respond differently to treatment than classic psychiatric disorders. ${ }^{24}$

\section{Temporal Lobe Epilepsy}

Epilepsy is a complex group of related neurological disorders involving unregulated 
nerve cell firing with a large variability in clinical presentation. Characteristically there is recurrent seizure activity. Temporal lobe epilepsy (TLE) is a subset of epilepsy known to present as a number of behavioral and neuropsychiatric complaints. Most presentations of TLE involve auras of emotional phenomena such as depression, fear, or anxiety, which can occur alone or with subsequent progression to complex partial or secondary generalized seizures. ${ }^{25}$ Many other bizarre presentations of TLE have been reported, including recurrent, potentially debilitating déjà vu, vivid recollection of past traumatic events mimicking posttraumatic stress disorder, paranoid delusions following olfactory triggers; and unprovoked attacks of depersonalization, derealization, anxiety, and dyspnea originally misdiagnosed as panic attack.

\section{Stroke}

The term "stroke chameleon" refers to presentations suggestive of other diseases that actually represent underlying strokes. Altered mental status is by far the largest block of these chameleons, with up to $30 \%$ of misdiagnosed strokes being misdiagnosed as altered mental status. The positive predictive value of altered mental status alone (ie, the chance that the diagnosis of altered mental status actually represents an undiagnosed acute stroke) is $7 \% .^{26}$

\section{Case Scenarios Continued}

\section{Case 1}

[The 58-year-old woman with intermittent chest pain.]

The patient's D-dimer and troponin I levels were normal. Before the EP had an opportunity to discuss the results and next steps with the patient, the nurse asked him to see the patient immediately. Upon entering her room, the EP noted that the patient appeared anxious. The patient said the shortness of breath had returned, and also that she felt as if she were "floating" off the gurney, outside of her body. A check of her vital signs revealed a heart rate of 106 beats/minute and blood pressure of 160/100 mm Hg. A repeat ECG was significant only for sinus tachycardia. In an effort to calm the patient, the EP reassured her that the ECG, chest X-ray, and screening laboratory studies were normal, and that there was no evidence of a heart attack. Relieved, the patient asked for an Ativan to calm her nerves. Upon further questioning, the patient sheepishly reported that she had been taking 3 to $6 \mathrm{mg}$ lorazepam for about 10 years, as prescribed by her family physician (FP) for anxiety. She further admitted that she abruptly discontinued taking the drug about one week before this ED visit after she'd heard on a daytime TV show that the medication was addictive.

After receiving lorazepam, the patient showed marked improvement. The EP's final impressions were atypical chest pain and acute panic attack precipitated by abrupt benzodiazepine withdrawal. After discussing the case with the patient's FP, the EP discharged the patient home with instructions to complete the cardiac evaluation as an outpatient. The EP also recommended that the patient resume taking lorazepam and follow-up with her FP within one week to discuss a benzodiazepine taper and alternative therapy for anxiety.

\section{Case 2}

[The 36-year-old woman with altered mental status.]

When the EP entered the patient's room, he witnessed the patient staring at her husband and striking him repetitively with her right arm. When the EP asked the patient to stop hitting, her husband told the EP that everything was alright and that the patient's neurologist had previously told them this behavior was caused by a seizure. While in the next examination room, one of the EP's colleagues had overheard some of the patient's history and recog- 
nized the name of the patient's neurologist as a specialist in partial complex seizures-one who had retired from the local medical school about 10 years ago.

After records from the local university hospital confirmed the patient's diagnosis of partial complex seizures, she was given intravenous lorazepam $2 \mathrm{mg}$; she became alert, conversational, and stopped flailing her right arm. She was then admitted to

\section{References}

1. Boyapati R, Papadopoulos G, Olver J, Geluk M, Johnson PD. An unusual presentation of herpes simplex virus encephalitis. Case Rep Med. 2012;241710.

2. Verma R, Anand KS. HIV presenting as youngonset dementia. I Int Assoc Provid AIDS Care. 2014;13(2):110-112.

3. Gheuens S, Pierone G, Peeters P, Koralnik IJ. Progressive multifocal leukoencephalopathy in individuals with minimal or occult immunosuppression. $J$ Neurol Neurosurg Psychiatry. 2010;81(3):247-254.

4. Sobhan T, Rowe HM, Ryan WG, Munoz C. Unusual case report: three cases of psychiatric manifestation of neurosyphilis. Psychiatr Serv. 2004;55(7):830-832.

5. Noblett J, Roberts E. The importance of not jumping to conclusions: syphilis as an organic cause of neurological, psychiatric and endocrine presentations. BMJ Case Rep. 2015;25:2015.

6. Ukwaja KN. Typhoid fever presenting as a depressive disorder-a case report. Rural Remote Health. 2010;10(2):1276.

7. Patra BN, Sharma A, Mehra A, Singh S. Complicated alcohol withdrawal presenting as self mutilation. $J$ Forensic Leg Med. 2014;21:46-47.

8. Stehman CR, Mycyk MB. A rational approach to the treatment of alcohol withdrawal in the ED. Am J Emerg Med. 2013;31(4):734-742.

9. Pétursson $\mathrm{H}$. The benzodiazepine withdrawal syndrome. Addiction. 1994;89(11):1455-1459.

10. Bosshart H. Withdrawal-induced delirium associated with a benzodiazepine switch: a case report. J Med Case Rep. 2011;5:207207.

11. Hassanian-Moghaddam H, Afzali S, Pooya A. Withdrawal syndrome caused by naltrexone in opioid abusers. Hum Exp Toxicol. 2014;33(6):561-567. doi:10.1177/0960327112450901

12. Hasin DS, Keyes KM, Alderson D, Wang S, Aharonovich E, Grant BF. Cannabis withdrawal in the United States: results from NESARC. J Clin Psychiatry. 69(9):1354-1363.

13. Okazaki Y. The epidemiology and pathogenesis of postpartum depression. Nihon Rinsho. 2001;59(8):1555-1559.

14. Sinert R, Su M, Secko M, Zehtabchi S. The utility of routine laboratory testing in hypoglycaemic emergency department patients. Emerg Med J. the hospital for medical stabilization of her frequent seizures.

Editor's Note: Part 2 of this article will appear in the June 2016 issue of Emergency Medicine and will cover psychiatric presentations related to dementia, cancer, cardiac disease, nutritional deficiencies, endocrine disorders, and toxins.

2009;26(1):28-31

15. Schneider P, Nejtek VA, Hurd CL. A case of mistaken identity: alcohol withdrawal, schizophrenia, or central pontine myelinolysis? Neuropsychiatr Dis Treat 2012;8:49-54.

16. Stojanovich L, Zandman-Goddard G, Pavlovich S, Sikanich N. Psychiatric manifestations in systemic lupus erythematosus. Autoimmun Rev. 2007;6(6):421-426.

17. Kayser MS, J Dalmau. Anti-NMDA receptor encephalitis, autoimmunity, and psychosis. Schizophr Res. 2014;pi:S0920-9964(14)00546-5

18. Tidswell J, Kleinig T, Ash D, Thompson P, Galletly C. Early recognition of anti-N-methyl D-aspartate (NMDA) receptor encephalitis presenting as acute psychosis. Australas Psychiatry. 2013;21(6):596-599.

19. Masopust J, Andrýs C, Bažant J, Vyšata O, Kuca K, Vališ M. Anti-NMDA receptor antibodies in patients with a first episode of schizophrenia. Neuropsychiatr Dis Treat. 2015;11:619-623.

20. de Cerqueira AC, Semionato de Andrade P, Godoy Barreiros JM, Teixeira AL, Nardi AE. Psychiatric disorders in patients with multiple sclerosis. Compr Psychiatry. 2015;63:10-14.

21. Mobbs RJ, Chandran KN, Newcombe RL. Psychiatric presentation of aneurysmal subarachnoid haemorrhage. ANZ I Surg. 2001;71(1):69-70.

22. Kar SK, Kumar D, Singh P, Upadhyay PK. Psychiatric manifestation of chronic subdural hematoma: the unfolding of mystery in a homeless patient. Indian J Psychol Med. 2015;37(2):239-242.

23. Nagel M, Rumpf HJ, Kasten M. Acute psychosis in a verified Huntington disease gene carrier with subtle motor signs: psychiatric criteria should be considered for the diagnosis. Gen Hosp Psychiatry. 2014;36(3):361.e3-e4.

24. Buoli M, Caldiroli A, Altamura AC. Psychiatric conditions in Parkinson disease: a comparison with classical psychiatric disorders. J Geriatr Psychiatry Neurol. 2016;29(2):72-91.

25. Bortz JJ. Neuropsychiatric and memory issues in epilepsy." Mayo Clin Proc. 2003;78(6):781-787.

26. Dupre CM, Libman R, Dupre SI, Katz JM, Rybinnik I, Kwiatkowski T. Stroke chameleons. J Stroke Cerebrovasc Dis. 2014;23(2):374-378. 
Editor's Note: The following is a list of additional sources of information about the medical mimics covered in this article.

\section{Acute Metabolic Conditions}

Schneider P, Nejtek VA, Hurd CL. A case of mistaken identity: alcohol withdrawal, schizophrenia, or central pontine myelinolysis? Neuropsychiatr Dis Treat. 2012;8:49-54.

Sinert R, Su M, Secko M, Zehtabchi S. The utility of routine laboratory testing in hypoglycaemic emergency department patients. Emerg Med J. 2009;26(1):28-31.

\section{Autoimmune Disorders}

Kayser MS, J Dalmau. Anti-NMDA receptor encephalitis, autoimmunity, and psychosis. Schizophr Res. 2014;pi:S09209964(14)00546-5.

Masopust J, Andrýs C, Bažant J, Vyšata $\mathrm{O}$, Kuca K, Vališ M. Anti-NMDA receptor antibodies in patients with a first episode of schizophrenia. Neuropsychiatr Dis Treat. 2015;11:619-623.

Stojanovich L, Zandman-Goddard G, Pavlovich S, Sikanich N. Psychiatric manifestations in systemic lupus erythematosus. Autoimmun Rev. 2007;6(6):421-426.

Tidswell J, Kleinig T, Ash D, Thompson P, Galletly C. Early recognition of anti-N-methyl $\mathrm{D}$-aspartate (NMDA) receptor encephalitis presenting as acute psychosis. Australas Psychiatry. 2013;21(6):596-599.

\section{Central Nervous Symptom Diseases}

Bortz JJ. Neuropsychiatric and memory issues in epilepsy. Mayo Clin Proc. 2003;78(6):781-787.

Buoli M, Caldiroli A, Altamura AC. Psychiatric conditions in Parkinson disease: a comparison with classical psychiatric disorders. J Geriatr Psychiatry Neurol. 2016;29(2):72-91. Deutsch SI, Rosse RB, Sud IM, Burket JA. Temporal lobe epilepsy confused with panic disorder: implications for treatment. Clin Neuropharmacol. 2009;32(3):160-162. Dupre CM, Libman R, Dupre SI, Katz JM, Rybinnik I, Kwiatkowski T. Stroke chameleons. J Stroke Cerebrovasc Dis. 2014;23(2):374-378.

Gandhi P, Ogunyemi B, MacDonald A, Gadit A. Psychosis in temporal lobe epilepsy: atypical presentation. BMJ Case Rep. 2012;18:2012.

Koubeissi MZ. Vivid recollection of a past traumatic event: a rare manifestation of temporal lobe seizures. Epileptic Disord. 2012;14(3):325-328.

Moulin CJ. The strange sensation of deja vu: not so strange in temporal lobe epilepsy. J Neurol Neurosurg Psychiatry. 2014;85(2):132.

Nagel M, Rumpf HJ, Kasten M. Acute psychosis in a verified Huntington disease gene carrier with subtle motor signs: psychiatric criteria should be considered for the diagnosis. Gen Hosp Psychiatry. 2014;36(3):361. e3-e4.

\section{Infections}

Blum JA, Neumayr AL, Hatz CF. Human African trypanosomiasis in endemic populations and travellers. Eur J Clin Microbiol Infect Dis. 2012;31(6):905-913.

Boyapati R, Papadopoulos G, Olver J, Geluk $M$, Johnson PD. An unusual presentation of herpes simplex virus encephalitis. Case Rep Med. 2012;241710.

Gerstenblith TA, Stern TA. Lyme disease: a review of its epidemiology, evaluation, and treatment. Psychosomatics. 2014;55(5):421429.

Gheuens S, Pierone G, Peeters P, Koralnik IJ. Progressive multifocal leukoencephalopathy in individuals with minimal or occult immunosuppression. J Neurol Neurosurg Psychiatry. 2010;81(3):247-254.

Noblett J, Roberts E. The importance of not jumping to conclusions: syphilis as an organic cause of neurological, psychiatric and endocrine presentations. BMJ Case Rep. 2015;25:2015.

Sobhan T, Rowe HM, Ryan WG, Munoz C. Unusual case report: three cases of psychiatric manifestations of neurosyphilis. Psychiatr Serv. 2004;55(7):830-832.

Ukwaja KN. Typhoid fever presenting as a depressive disorder - a case report. Rural Remote Health. 2010;10(2):1276.

Verma R, Anand KS. HIV presenting as young-onset dementia. J Int Assoc Provid AIDS Care. 2014;13(2):110-112.

Verma A, Kumar A. Neurocysticercosis presenting as acute psychosis: a rare case report from rural India. Asian J Psychiatr. 2013;6(6):611-613.

Zamora CA, Nauen D, Hynecek R, et al. Delayed posthypoxic leukoencephalopathy: a case series and review of the literature. Brain Behav. 2015;5(8):e00364.

\section{Multiple Sclerosis}

Agan K, Gunal DI, Afsar N, Tuncer N, Kuscu K. Psychotic depression: a peculiar presentation for multiple sclerosis. Int J Neurosci. 2009;119(11):2124-2130.

Aggarwal A, Sharma D, Kumar R, Sharma R. Acute psychosis as the initial presentation of MS: a case report. Int MS J. 2011;17(2):5457.

de Cerqueira AC, Semionato de Andrade P, Godoy Barreiros JM, Teixeira AL, Nardi AE. Psychiatric disorders in patients with multiple sclerosis. Compr Psychiatry. 2015;63:10-14. Mendonça MD, Leitão C, Barahona-Corrêa B, Lampreia T. Paroxetine-related adult-onset tic disorder: a presentation of primary progressive multiple sclerosis. J Neuropsychiatry Clin Neurosci. 2015;27(3):e214-e215

\section{Pharmacological Withdrawal} Syndromes

Armstrong J, Little M, Murray L. Emergency department presentations of naltrexoneaccelerated detoxification. Acad Emerg Med. 2003;10(8):860-866.

Bosshart $\mathrm{H}$. Withdrawal-induced delirium associated with a benzodiazepine switch: a case report. J Med Case Rep. 2011;5:207207.

Findley, JC, Joshi KG. A case of repeated brief psychotic episodes secondary to discontinuation of non-prescription estrogen replacement therapy. Psychosomatics. 2012;53(3):285-288.

Hasin DS, Keyes KM, Alderson D, Wang S, Aharonovich E, Grant BF. Cannabis withdrawal in the United States: results from NESARC. J Clin Psychiatry. 2008;69(9): 1354-1363.

Hassanian-Moghaddam H, Afzali S, Pooya A. Withdrawal syndrome caused by naltrexone in opioid abusers. Hum Exp Toxicol. 2014;33(6):561-567.

Huber TJ, Nickel V, Tröger M, Schneider $\mathrm{U}$, Husstedt H, Emrich HM. A syndrome of psychosis following discontinuation of an estrogen-progestogen contraceptive and improvement following replacement: A case report. Neuropsychobiology. 1999;40(2): 75-77.

Mahé V, Dumaine A. Oestrogen withdrawal associated psychoses. Acta Psychiatr Scand. 2001104(5):323-331.

Okazaki Y. The epidemiology and pathogenesis of postpartum depression. Nihon Rinsho. 2001;59(8):1555-1559.

Patra BN, Sharma A, Mehra A, Singh S. Complicated alcohol withdrawal presenting as self mutilation. J Forensic Leg Med. 2014:21:46-47.

Pétursson $\mathrm{H}$. The benzodiazepine withdrawal syndrome. Addiction. 1994;89(11):1455-1459. Stehman CR, Mycyk MB. A rational approach to the treatment of alcohol withdrawal in the ED. Am J Emerg Med. 2013;31(4):734-742.

\section{Trauma}

Hayhow B, Velakoulis D, Dewhurst R, Gaillard F. Neuropsychiatric presentation following acute hypoxic-ischaemic encephalopathy. Aust N Z J Psychiatry. 2015;49(2):188-189. James BO, Omoaregba JO, Lawani AO, Ikeji $\mathrm{CO}$, Igbinowanhia NG. Subdural haematoma presenting as catatonia in a 20-year-old male: a case report. Cases J. 2009;2:8032. Kar SK, Kumar D, Singh P, Upadhyay PK. Psychiatric manifestation of chronic subdural hematoma: the unfolding of mystery in a homeless patient. Indian J Psychol Med. 2015;37(2):239-242.

Mobbs RJ, Chandran KN, Newcombe RL. Psychiatric presentation of aneurysmal subarachnoid haemorrhage. ANZ J Surg. 2001;71(1):69-70. 